\title{
A multiple-site-specific heteroduplex tracking assay as a tool for the study of viral population dynamics
}

\author{
Wolfgang Resch*, Neil Parkin ${ }^{\ddagger}$, Erin L. Stuelke*, Terri Watkins*, and Ronald Swanstrom* ${ }^{* \S}$ \\ *University of North Carolina Center for AIDS Research and 'Departments of Biochemistry and Biophysics, University of North Carolina, \\ Chapel Hill, NC 27599; and ₹ViroLogic, Inc., 270 East Grand Avenue, South San Francisco, CA 94080 \\ Communicated by Clyde A. Hutchison III, University of North Carolina, Chapel Hill, NC, October 26, 2000 (received for review March 16, 2000)
}

\begin{abstract}
Rapidly evolving entities, such as viruses, can undergo complex genetic changes in the face of strong selective pressure. We have developed a modified heteroduplex tracking assay (HTA) capable of detecting the presence of single, specific mutations or sets of linked mutations. The initial application of this approach, termed multiple-site-specific (MSS) HTA, was directed toward the detection of mutations in the HIV-1 pro gene at positions $46,48,54,82$, 84 , and 90 , which are associated with resistance to multiple protease inhibitors. We demonstrate that MSS HTA is sensitive and largely specific to all targeted mutations. The assay allows the accurate and reproducible quantitation of viral subpopulations comprising $3 \%$ or more of the total population. Furthermore, we used MSS HTA in longitudinal studies of pro gene evolution in vitro and in vivo. In the examples shown here, populations turned over rapidly and more than one population was present frequently. To demonstrate the versatility of MSS HTA, we also constructed a probe sensitive to changes at positions 181 and 184 of the RT coding domain. Changes at these positions are involved in resistance to nevirapine and 2', $3^{\prime}$-dideoxy-3'-thiacytidine (3TC), respectively. This assay easily detected the evolution of resistance to 3TC. MSS HTA provides a rapid and sensitive approach for detecting the presence of and quantifying complex mixtures of distinct genotypes, including genetically linked mutations, and, as one example, represents a useful tool for following the evolution of drug resistance during failure of HIV-1 antiviral therapy.
\end{abstract}

O ne important example of the adaptive potential of HIV-1 is the evolution of strains resistant to protease or reverse transcriptase (RT) inhibitors. The population dynamics underlying the evolution of resistance are poorly understood, mostly because of limitations imposed by the methods available. The cloning and sequencing of PCR products from viral RNA or proviral DNA is one approach that is used widely to study changes in viral subpopulations. Although this method can yield detailed data about the sequences present, it is labor intensive. Moreover, the number of clones that are needed to determine accurately the frequency of distinct sequence subpopulations is large. A number of nonsequencing methods, such as the Line Probe Assay (1), sequence-specific PCR (2), molecular beacons (3), oligonucleotide ligation assays $(4,5)$, bulk sequencing (6), or others $(7,8)$, can be used to determine genotypes and to estimate the frequency of populations differing at defined positions. Although these techniques are easier than sequencing multiple clones, some have limited sensitivity, are only capable of detecting individual mutations, or cannot establish the linkage of detected mutations in the genome of a subpopulation.

The heteroduplex tracking assay (HTA) can be used to study viral population dynamics. In this assay, a radioactively labeled probe is annealed to a PCR product (derived from the total viral population) to generate probe-PCR product heteroduplexes that can be separated by gel electrophoresis. Clustered mutations, insertions, or deletions result in altered migration of the heteroduplex, which can reveal distinct subpopulations of viral genomes. HTA is quantitative, sensitive, simple, and allows the inference of linkage between different mutations. The standard HTA, because of its reliance on naturally occurring clustered point mutations, insertions, and deletions to induce altered heteroduplex migration, has been restricted largely to the investigation of the highly variable envelope region of HIV and other viruses $(9-12)$.

Here, we describe a multiple-site-specific (MSS) HTA that allows the detection of multiple predefined point mutations. The specificity for detecting point mutations is introduced into the probe by changing nucleotides close to the sites of interest. This is an application of the universal heteroduplex generator technology (13-15) but altered to detect viral subpopulations with varyious numbers of specific mutations. We have generated a probe capable of detecting mutations at positions $46,48,54,82$, 84 , and 90 of the HIV-1 protease gene (pro). These six mutations contribute to resistance to the HIV-1 protease inhibitors ritonavir, saquinavir, and indinavir (reviewed in ref. 16). We show examples of the evolution of HIV-1 measured by MSS HTA under the selective pressure of protease inhibitors in vitro and in vivo. Finally, this strategy has been extended to detect mutations in the HIV-1 DNA polymerase gene ( $p o l)$ associated with resistance to inhibitors of RT.

\section{Materials and Methods}

Patient Samples. Blood plasma samples were obtained—unlinked to patient identifiers-as excess tissue samples with institutional review board approval.

Plasmids and Probes. A 247-bp fragment of pro was amplified from the HIV-1 molecular clone Hxb-2R with a wild-type pro sequence (17) by using PCR with primers PRAMPUP (5'AACTAAAGGAAGCTCTATTAGATACAGGAG- ${ }^{\prime}$ ) and PRAMPDW (5'-GGAAAATTTAAAGTGCAACCAATCTGA-3'). The amplified product spanned codons $18-99$ of pro. The PCR product was cloned into pT7Blue (Novagen). The plasmid pPR-EB was generated by moving the EcoRI in the multiple cloning site closer to the inserted pro fragment for improved labeling.

A 166-bp fragment of HIV-1 molecular clone Hxb-2r pol gene spanning RT codon positions 150 through 205 was cloned into pT7blue analogously (plasmid pRTWT). The fragment was amplified by using primers RTUP1 (5'-CACAGGGATGGAAAGGATCACCA-3') and RTDOWN1 (5'-AGCTCCTCTATTTTTGTTCTATGCTGC-3').

Abbreviations: HIV-1, HIV type 1; MSS, multiple-site-specific; HTA, heteroduplex tracking assay; RT, HIV-1 reverse transcriptase; 3TC, 2',3'-dideoxy-3'-thiacytidine.

§To whom reprint requests should be addressed at: University of North Carolina Center for AIDS Research, CB 7295, Chapel Hill, NC 27599-7295. E-mail: risunc@med.unc.edu.

The publication costs of this article were defrayed in part by page charge payment. This article must therefore be hereby marked "advertisement" in accordance with 18 U.S.C. $\S 1734$ solely to indicate this fact.

Article published online before print: Proc. Natl. Acad. Sci. USA, 10.1073/pnas.011511298. Article and publication date are at www.pnas.org/cgi/doi/10.1073/pnas.011511298 
Site-Directed Mutagenesis. Site-directed mutagenesis was done as described (18). The mutagenic primers used for the site-directed mutagenesis of the pro fragment in pPR-EB were $47 \mathrm{~b}\left(5^{\prime}-\right.$ AATTCCCCTTATCCTTTTTG-3'), 90a (5'-AATCTGAGTANANAGATTTC-3'), 54d (5'-CTGTCTTACTTNNATNNAACCTCCA-3'), 82/84d2 (5'-AGAAAGATTTCTTCCAA $\{$ TG $\}\{$ TG $\}$ \{TC $\}$ T $\{$ TG $\}$ TA $\{$ TA $\}$ AC $\{$ AC $\}$ GGTGTAGGTCCTACTAA-3' ${ }^{\prime}$. Primers used in the site-directed mutagenesis of pRTWT were pESA (5'-ATACAAATCA $\{$ TA $\}\{$ GCT $\}$ C $\{$ TA $\}$ T $\{$ GAT $\}$ TATTGATAGAT-3') and pESB (5'-ATGCTTATAT $\{$ TC $\}$ G $\{$ GC $\}$ T$\{$ TCG $\}$ G $\{$ AT $\}$ TAACTATGTC-3' ${ }^{\prime}$. The Ns represent the inclusion of all four nucleotides at that position in the oligonucleotide synthesis, and the bracketed nucleotides represent the inclusion of a subset of the bases at that position during synthesis.

Probe Labeling. A $10 \mu \mathrm{g}$ aliquot of pPR-EB6.1 was digested with EcoRI followed by the incorporation of $\left[\alpha-{ }^{35} \mathrm{~S}\right] \mathrm{dATP}(0.1 \mathrm{mCi}$, $3000 \mathrm{mCi} / \mathrm{mmol}$ ) in a fill-in reaction using the Klenow fragment of DNA polymerase I (10 units). After incubation for $15 \mathrm{~min}$ at room temperature, the reaction was stopped by the addition of EDTA and the enzymes were inactivated by heat. The probe was released from the plasmid by $N d e$ I digestion in the same mixture. Unincorporated nucleotides were removed by column purification over a MicroSpin G50 spin column (Amersham Pharmacia).

Plasmid pRT2.1 was labeled analogously, except that BamHI was used for the first digestion and unlabeled dGTP was added to the labeling reaction to fill in the first position in the $5^{\prime}$ overhang.

Sequencing of Patient-Derived pro Clones. Separate RT-PCR products were generated to determine the pro sequences of clones from patient 1005 . The primers used (5'-GGGCCATCCATTCCTGGC-3' and 5'-CAGAGCCAACAGCCCCAC-3') amplify a fragment from the gag p6 coding region to the $5^{\prime}$ end of pol. The RT-PCR products were cloned into pT7Blue as described previously and were sequenced by using dye-terminator chemistry. Molecular clones were assigned to the bands found in the patient by using HTA, and several clones were sequenced for each HTA band except the lower band at month 9 .

HTA. Heteroduplexes were generated in annealing buffer (100 $\mathrm{mM} \mathrm{NaCl} / 10 \mathrm{mM}$ Tris $\cdot \mathrm{HCl}, \mathrm{pH}$ 7.5/2 mM EDTA) by denaturing a mixture of $5 \mu \mathrm{l}$ of $\mathrm{PCR}$ product and $0.1 \mu \mathrm{g}$ of radioactively labeled probe for $2 \mathrm{~min}$ at $95^{\circ} \mathrm{C}$, followed by annealing during cooling for $5 \mathrm{~min}$ at room temperature. The heteroduplexes were subjected to $12 \%$ PAGE (acrylamide:bisacrylamide $=61.5: 1$, $\mathrm{vol} / \mathrm{vol}$ ) in a GIBCO/BRL gel apparatus VP-16-2 at $15 \mathrm{~mA}$ per gel in TBE gel buffer. After separation, the heteroduplexes were visualized by autoradiography. Quantitative analysis was performed with a Storm 840 phosphor imager by using the IMAGEQUANT software (Molecular Dynamics). When this assay was done with two unlabeled PCR fragments, the heteroduplexes were visualized by silver stain (Bio-Rad).

The mobility $(k)$ of a heteroduplex was calculated as the distance between the heteroduplex and the top of the gel divided by the distance between the double-stranded probe and the top of the gel.

RNA Isolation and RT-PCR. Viral RNA was isolated from $140 \mu \mathrm{l}$ of blood plasma by using the QIAmp Viral RNA kit (Qiagen, Chatsworth, CA). For the pro fragment, reverse transcription was carried out with the Titan One Tube RT-PCR System (Roche Molecular Biochemicals) with the following modifications: RT reactions contained $5 \mu$ l of RNA solution, $1 \times$ Titan RT-PCR buffer, $5.25 \mathrm{mM} \mathrm{MgCl}_{2}, 0.5 \mathrm{mM}$ of each dNTP, $5 \mathrm{mM}$ DTT, $2 \mu \mathrm{M}$ primer PRAMPDW, 20 units of RNase inhibitor (Roche Molecular Biochemicals), and 12.5 units of avian myeloblastosis virus RT (Roche Molecular Biochemicals) in a final volume of $20 \mu \mathrm{l}$. Reactions were carried out at $42^{\circ} \mathrm{C}$ for $45 \mathrm{~min}$ followed by heat inactivation. PCR mix $(30 \mu \mathrm{l} ; 1 \times$ Titan RT-PCR buffer, $5 \mathrm{mM}$ DTT, $1.3 \mu \mathrm{M}$ primer PRAMPUP, and $1 \mu$ l of Titan enzyme mix) was added. PCR was performed in a Stratagene 40 Robocycler $\left(1\right.$ cycle at $95^{\circ} \mathrm{C}$ for $3 \mathrm{~min}, 56^{\circ} \mathrm{C}$ for $60 \mathrm{~s}$, and $68^{\circ} \mathrm{C}$ for $60 \mathrm{~s} ; 40$ cycles at $95^{\circ} \mathrm{C}$ for $40 \mathrm{~s}, 56^{\circ} \mathrm{C}$ for $60 \mathrm{~s}$, and $68^{\circ} \mathrm{C}$ for $60 \mathrm{~s}$ to $4 \mathrm{~min}$ ). Extension times were increased by $1 \mathrm{~min}$ per 10 cycles from $60 \mathrm{~s}$ to $4 \mathrm{~min}$.

The RT fragment was amplified analogously, except for a lower $\mathrm{Mg}^{2+}$ concentration of $1.5 \mathrm{mM}$ during reverse transcription and altered cycling conditions $\left(1\right.$ cycle at $95^{\circ} \mathrm{C}$ for $3 \mathrm{~min}$, $50^{\circ} \mathrm{C}$ for $60 \mathrm{~s}$, and $68^{\circ} \mathrm{C}$ for $60 \mathrm{~s} ; 40$ cycles at $95^{\circ} \mathrm{C}$ for $45 \mathrm{~s}, 50^{\circ} \mathrm{C}$ for $60 \mathrm{~s}$, and $68^{\circ} \mathrm{C}$ for $60 \mathrm{~s}$ to $4 \mathrm{~min}$ ).

DNA Isolation and Genomic PCR. HIV-1 cultures for in vitro selection of protease inhibitor resistance, genomic DNA isolation, and PCR amplification for bulk sequencing were described (19). PCR for MSS HTA was done by using the Expand High Fidelity PCR System (Roche Molecular Biochemicals) with the following modifications: reactions contained $0.2-4 \mu \mathrm{g}$ of total cellular DNA, $1 \times$ Titan RT-PCR buffer, $3 \mathrm{mM} \mathrm{MgCl} 2,0.2 \mathrm{mM}$ of each dNTP, $5 \mathrm{mM}$ DTT, $0.5 \mu \mathrm{M}$ primers PRAMPUP and PRAMPDW, and $1 \mu \mathrm{l}$ of Expand enzyme mix. Cycling conditions were as described previously.

Drug Susceptibility Assay and Bulk Sequencing of pro. The bulk sequence of pro was determined as described (20). Drug susceptibility of viral populations was measured by using a recombinant virus assay (20).

\section{Results}

Modification of an HTA Probe to Detect Multiple Point Mutations. An MSS HTA probe that was sensitive to base changes at six different positions in the subgroup B HIV-1 pro gene was generated by multiple rounds of site-directed mutagenesis followed by screens for appropriate mutations. To generate a probe with sensitivity to a specific base change, libraries of mutant clones with base changes in close proximity to the target mutation were generated. Clones of this library were then amplified and the PCR products were annealed to either wildtype or mutant PCR products. The heteroduplexes were separated by PAGE, and a clone with a differential mobility change between wild-type and mutant PCR products was chosen. Sensitivity to additional mutations was introduced by repeating the mutagenesis-and-screening cycle. For the pro MSS probe 6.1, 12 bases were modified to achieve sensitivity to changes at six positions (Fig. 1A). This probe, when radioactively labeled and annealed to the sequences containing the targeted mutations, displayed slower mobility in PAGE than the probe wild-type heteroduplex (Fig. 1B). The presence of multiple mutations resulted frequently in a change of the mobility of the heteroduplex when compared with single mutations. This observation implies that the detection of multiple bands in a sample indicates the presence of distinct genotypes that differ by sets of linked mutations. In the absence of sequence information, the nature of the linked sequences cannot be known but their presence is inferred by the detection of distinct species.

MSS HTA Is Specific Largely for Changes in the Region of the Targeted Positions. To assess the specificity of MSS HTA for the resistance-relevant mutations, we tested the MSS probe 6.1 against a panel of mutated protease-coding domains. This panel contained clones with only single nucleotide changes and covered most pro codons between positions 20 and 95 (21). The sequences were amplified from M13 phage stocks with mutated HIV-1 pro-pol inserts, and the PCR products were subjected to MSS HTA. The mobility of the heteroduplexes is shown graphically in Fig. 2. Peaks representing heteroduplexes of decreased 


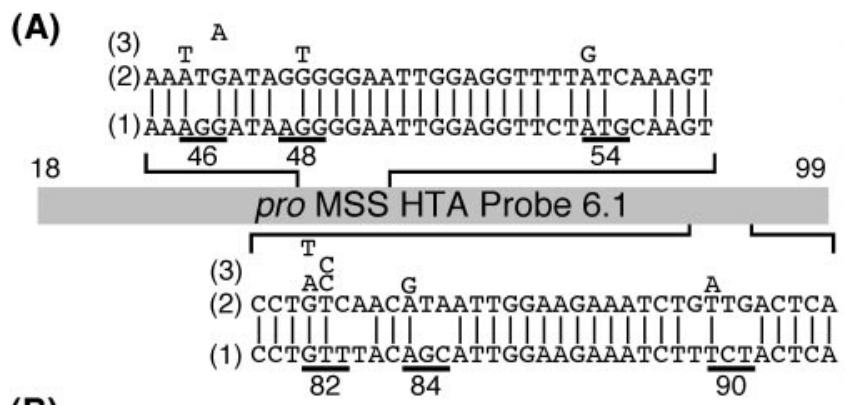

(B)

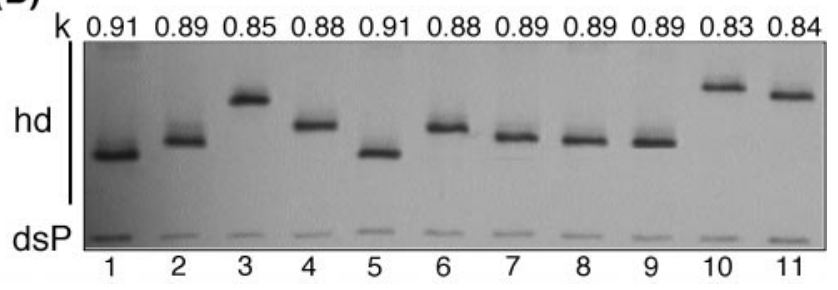

Fig. 1. Characteristics of the pro MSS HTA probe 6.1. (A) Sequence of the probe (1) aligned to the $\mathrm{Hxb}-2 \mathrm{r}$ sequence (2) in the areas of the target positions. Base changes leading to resistance are indicated above the wildtype sequence (3). Resistance-relevant changes are in close proximity to probe wild-type mismatches. $(B)$ Mobility of the radioactively labeled probe annealed to PCR products of pro genes with point mutations. Only the heteroduplexes (hd) and the probe that annealed to its fully complementary strand (double-stranded probe, dsP) are shown. Lanes: 1, wild type; 2, M46I; 3, G48V; 4, I54T; 5, L63P; 6, V82T; 7, V82A; 8, I84V; 9, L90M; 10, G48V/V82T; and 11, $\mathrm{G} 48 \mathrm{~V} / \mathrm{L90M}$. The mobility $(k)$ of each hd relative to the dsP is indicated above all lanes. Note that wild type and L63P, a nontargeted mutation, have identical mobilities, whereas all of the targeted mutations display lower mobilities. The mobilities of the hds are calculated relative to the dsP to control for differences in the gel or in the electric field between lanes and gels.

mobility were found at all targeted positions. As would be expected, some of the changes in codons adjacent to targeted positions resulted in mobility shifts as well, reducing the specificity of the MSS HTA. This regional sensitivity can be seen with

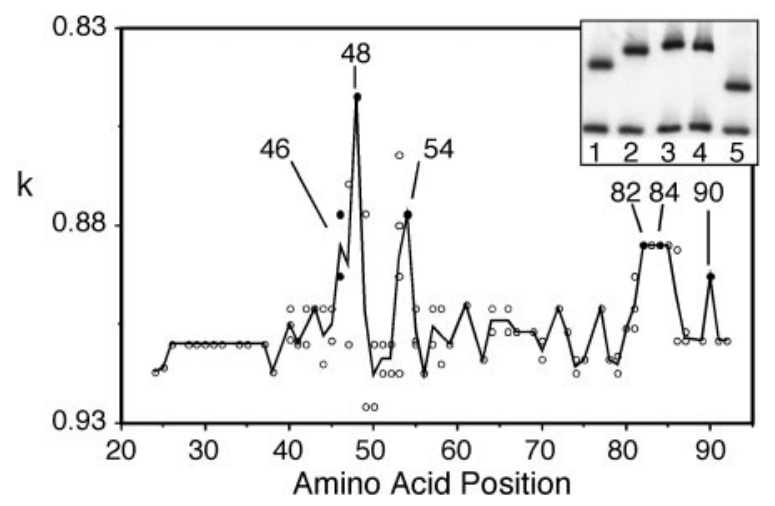

Fig. 2. Specificity of the pro MSS HTA probe. $(A)$ Mobility $(k)$ of a panel of pro genes containing different point mutations annealed to the pro MSS probe plotted against the codon position of the mutation. Resistance-relevant mutations are denoted with $(\bullet)$, all other mutations with $(\bigcirc)$. The line represents the average of all mutations tested except for the resistance-relevant positions (indicated) where only resistance-associated mutations were averaged. This was necessary because in some cases, nonresistance mutations at these positions had higher mobilities than resistance-associated mutations. The six targeted mutations had the lowest mobilities. However, some mutations in codons adjacent to resistance-relevant positions induce mobility changes as well. The gel shows an example of five point mutations encoding the following substitutions: lane 1, 147R; lane 2, G48V; lane 3, G48E; lane 4, G48A; and lane 5, G49G. substitutions encoding I47R, G49V, F53Y, F53V, and silent mutations at positions 83 and 85 , which resulted in mobility shifts similar to those induced by the targeted mutations. However, changes at more distal, nontargeted positions do not cause significant mobility changes. In addition, only a total of 19 coding changes in the 10 positions adjacent to the targeted positions were found in 709 unrelated pro sequences from untreated individuals retrieved from an HIV-1 protease database (22). This low degree of variability in the flanking codons reduces the impact of the regional sensitivity of the probe when assessing the presence of resistance-associated mutations.

MSS HTA Allows the Accurate Quantitation of HIV-1 Populations. We determined that the HTA band intensities were an accurate reflection of the PCR mixture by creating mixtures of PCR products amplified separately from different pro genes. Two different mixtures were analyzed to demonstrate sequence independence of the assay. To generate both mixtures, a wild-type PCR product was combined with a PCR product containing either 8 or 12 mutations. We used a high number of changes to confirm that the annealing reaction was not biased toward sequences with fewer mismatches. The products were mixed at various ratios and the mixtures were diluted over a 100 -fold range so that the subsequent annealing reaction would span conditions from excess target to excess probe. The abundance of the PCR products in the mixture was measured by MSS HTA and showed a linear correlation with the known input abundance at all dilutions (Fig. 3A). The best-fit line does not intersect the origin, probably because of a small inaccuracy in the initial determination of the concentration of PCR products. The linear relation was independent of whether probe or target molecules were in excess, and MSS HTA accurately quantified the minority population down to $3 \%$ of the total. No input levels below $3 \%$ were tested because the number of template molecules typically available in clinical samples precluded the reproducible quantitation (i.e., sampling) of viral variants with lower than a $3 \%$ frequency. Also, a species that represents $3 \%$ of the total population is well above the background that becomes apparent with longer exposure of the gel.

The question of PCR recombination was addressed by amplifying mixtures of viral molecular clones. Products of recombination during PCR would be expected to shift differently from either of the two parental sequences, and would therefore be visible as a new band or as a smear between the two parental bands. However, no such bands were detected in our reconstruction experiments, indicating that recombination during PCR does not significantly impact the results of MSS HTA (data not shown).

To validate that a viral population was sampled correctly by RT-PCR, the amplification reactions were replicated to compare HTA patterns. Reproducible HTA patterns can be achieved only if the population has been accurately sampled, because stochastic events under conditions of limiting templates during PCR would not allow the reproduction of HTA patterns if the sampling of the viral population was incomplete. Reproducible HTA patterns from clinical samples are typically observed with as few as 2,000 RNA template molecules per reaction (data not shown). Moreover, we amplified known mixtures of different pro genes to examine the minimal number of DNA templates needed for the accurate quantitation of a pro population comprising $10 \%$ of the total (Fig. 3B). We found that 1,200 DNA template molecules were sufficient to quantitate a subpopulation representing $10 \%$ of the total population reproducibly.

Correlation Between MSS HTA and Drug Susceptibility. MSS HTA was performed on RT-PCR products derived from viral RNA that was isolated from 21 patient plasma samples and correlated with reduced sensitivity to HIV-1 protease inhibitors, as was 
(A)

(B)
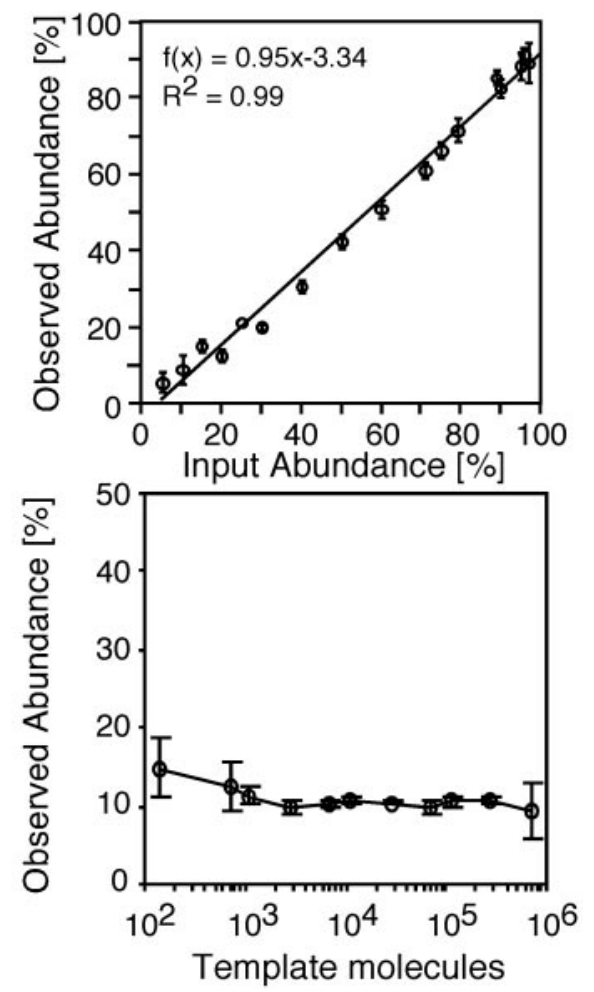

Fig. 3. Reproducibility and sensitivity of the pro MSS HTA. (A) The MSS HTA reflects the populations in the PCR product accurately and reproducibly at high template numbers. PCR products of two pro genes were mixed at known ratios; the mixtures were then diluted over a 100 -fold range and annealed to the MSS HTA probe 6.1. The abundance of one of the products was determined by using the MSS HTA and compared with the known abundance. Error bars represent standard deviations from 5-9 experiments. (B) Amplification of pro gene mixtures. Mixtures of pro genes were amplified and quantified by using the MSS HTA. The smallest number of templates required to detect and quantify a $10 \%$ population reproducibly is 1,200 . Error bars are the standard deviation from three experiments. The sequences used encoded the following changes from Hxb: (1) T12S, K43R, M46I, Q61L, L63P, V82A; (2) T12S, K43R, M46I, I54V, Q61H, L63P, V82F.

determined by a recombinant virus assay. The samples were selected based on phenotypic evidence of resistance in protease inhibitor-experienced subjects. The average reduction in susceptibility to ritonavir, indinavir, and saquinavir $\left(r_{\mathrm{a}}\right)$ of viral populations compared with the molecular clone NL4-3 was correlated with the mobility $(k)$ of the sample. Of the 21 subjects examined, 11 harbored multiple pro populations (Fig. $4 A$ ). In these cases the mobility ratio of the most abundant population was used. The amino acid changes found in the bulk sequence of the viral populations are shown in Fig. $4 A$ as well. To identify the baseline variability of $k$, seven samples from protease inhibitor-naïve patients were included in the analysis and are shown in Fig. $4 B$ together with the mobility of the molecular clones NL4-3 and Hxb-2r. Unlike any of the 21 samples from the protease inhibitor-experienced patients, seven of these nine controls had mobilities greater than 0.89 (Fig. 4B). In good agreement with the reduced probe mobility, 20 of the 21 protease inhibitor-experienced patients harbored viral sequences that conferred a greater than 2.5 -fold reduction of average drug susceptibility, and all viral populations contained at least one of the mutations targeted by the MSS HTA probe. Thus, the pro MSS HTA can readily detect the presence of reduced susceptibility markers to the target protease inhibitors, but, as can be seen from Fig. 4B, the mobility ratios do not show a clear linear correlation with the level of
(A)

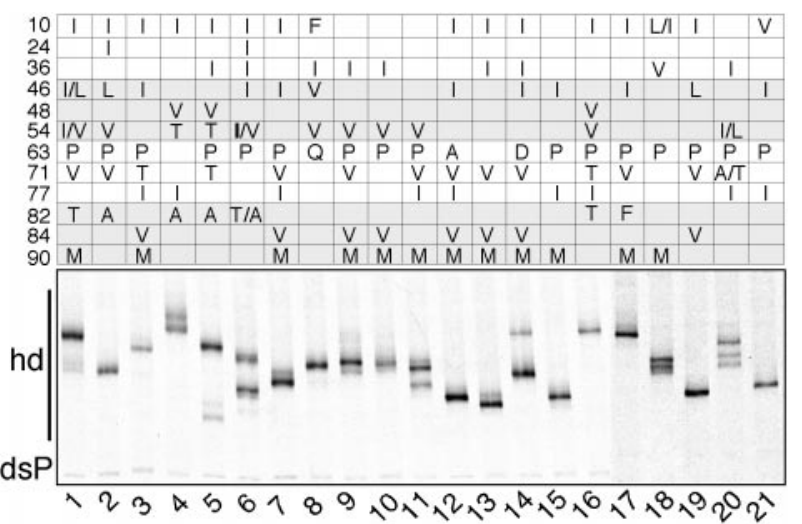

(B)

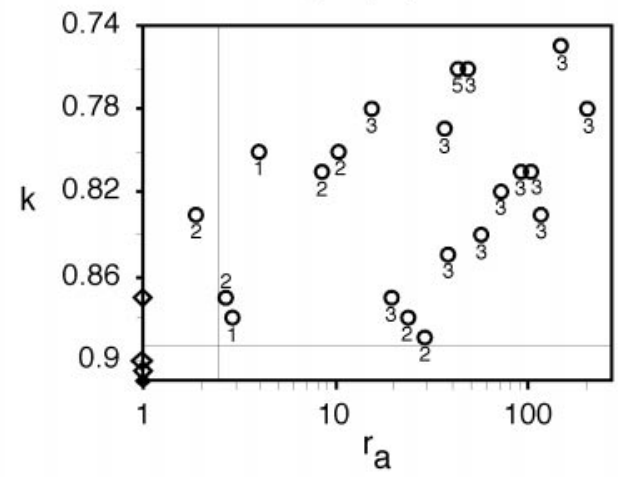

Fig. 4. Cross-sectional study of viral pro populations and correlation of MSS HTA mobility shifts with reduced drug susceptibility. (A) The MSS HTA analysis of RT-PCR products from 21 patient plasma samples with different treatment histories. Differences in the bulk sequence of the RT-PCR products from HIV-1 clade $B$ consensus are shown above the gel. Two amino acids indicate a mixed population. Note that positions that were not resistance-associated were omitted. Note also that each population contains at least one of the targeted mutations of the MSS HTA probe (highlighted in gray). In 11/21 cases, multiple populations differing at or near the targeted positions were found by HTA, whereas population-based sequencing identified mixed populations in only 4 subjects. hd, heteroduplex; dsP, double-stranded probe. $(B)$ Mobility $(k)$ of the most prominent MSS HTA band of each subject correlated with the average reduction of susceptibility to ritonavir, saquinavir, and indinavir $\left(r_{\mathrm{a}}\right)$ of the complete virus population compared with molecular clone NL4-3 (O). The labels indicate the number of targeted mutations seen in the bulk sequence. For comparison, the mobility of bands corresponding to viral populations from seven protease inhibitor-naïve patients $(\diamond)$ and the mobility of molecular clones NL4-3 and $\mathrm{Hxb}-2 \mathrm{r}(\bullet)$ are shown. Not all of these are visible on the plot, because some mobilities are identical.

susceptibility. The weak correlation between mobility and the level of susceptibility may be ascribed to the absence of a strict correlation between mobility change and the magnitude of resistance conferred by individual mutations. Similarly, the additive effects of multiple mutations on mobility and resistance are not likely to be strictly correlated. In addition, mobility can be affected by mutations in positions adjacent to the resistance mutations. Finally, any combination of mutations not targeted by the probe, or outside the region covered by the probe that would contribute to a resistance phenotype, would go undetected. These complicating factors limit the utility of MSS HTA in identifying complex patterns of pro resistance mutations in a cross-sectional analysis. The usefulness of MSS HTA for longitudinal studies, however, is affected less, because the main focus of longitudinal studies is the emergence of new viral variants rather than the exact correlation of the mobility ratio of specific variants with their phenotype. Note that in this type of analysis, MSS HTA complements and focuses the more labor-intensive approach of sequence determination. 
(A)

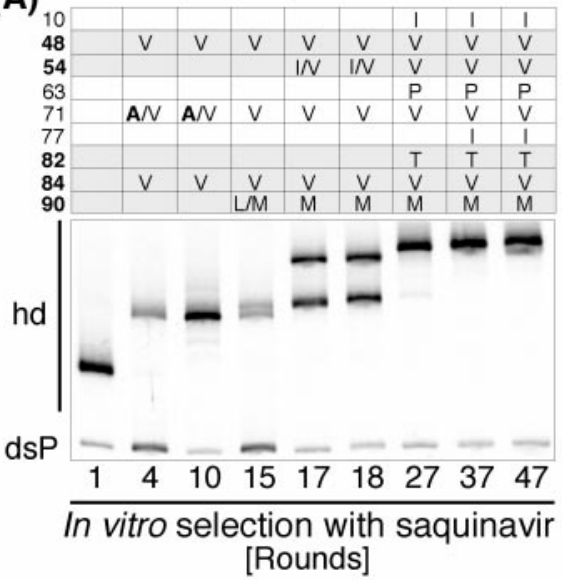

(B)

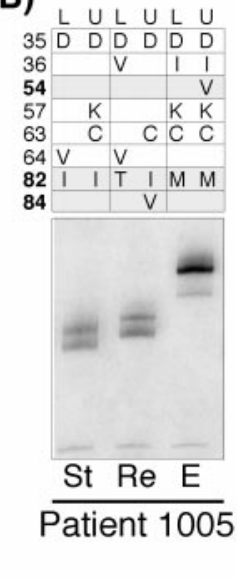

Fig. 5. Longitudinal study of viral pro subpopulations under the selective pressure of protease inhibitors in vitro and in vivo. (A) The MSS HTA of nine different passages of a virus culture that was subjected to increasing concentrations of saquinavir. The stepwise shift of the MSS HTA bands to higher positions can be seen and this shift correlates with an increasing number of resistance mutations seen in bulk sequences derived from proviral DNA. Positions highlighted in gray are target positions of the MSS probe. Two amino acids indicate a mixed population. hd, heteroduplex; dsP, double-stranded probe. (B) Patient 1005 was followed during a trial of ritonavir from the start of monotherapy (St, day 1 ), through to the first timepoint of rebound of viral RNA after initial response ( $R e$, day 114), to the endpoint of the trial $(E$, day 228). The pro sequences of molecular clones corresponding to different populations are indicated ( $L$, lower band; $U$, upper band). Again, the target positions of the MSS HTA probe are highlighted in gray. Note that the L63C mutation represents a change of three nucleotides (CTC to TGT) which likely leads to a mobility shift.

Longitudinal Studies of HIV-1 Evolution in Vitro and in Vivo by Using MSS HTA. MSS HTA was applied to viral populations in cell culture during passage with increasing selective pressure from saquinavir (Fig. 5A). The selection gave rise to sequence changes that were detected as HTA bands with slower migration. The evolution of resistance appears to be a stepwise process in the selected cultures with several populations coexisting at multiple times. Sequence analysis confirmed that the observed mobility changes coincided with the accumulation of resistance mutations (Fig. 5A). This experiment also demonstrates that the MSS HTA resolves different mixtures of linked mutations. Similar results were obtained when selections were carried out with other protease inhibitors (T.W. and R.S., unpublished observation).

MSS HTA was also able to separate viral populations present in vivo and the populations could be followed over time. As an example, we examined plasma samples from an HIV-1-infected subject who participated in a ritonavir clinical trial (23). When ritonavir was added to this subject's existing RT inhibitor therapy, viral load decreased by approximately 100 -fold, rebounded within 3 months to pretreatment levels, and remained stable for 6 months in the continued presence of ritonavir. As shown in Fig. $5 B$, the pro variants in this subject's blood plasma, when represented as MSS HTA bands, shifted to successively higher positions over time. At the start of ritonavir therapy (St), the rebound $(\mathrm{Re})$, and the end $(\mathrm{E})$, there were two pro sequences present. Again, sequence analysis confirmed that the changing mobility of the bands was caused by resistance mutations (Fig. $5 B$ ). In addition, a change of all 3 nucleotides of codon 63 (L63C) contributed to the mobility shifts. A similar pattern was observed for 11 of the 12 patients who were studied during this trial (W.R. and R.S., unpublished observation).

Adaptation of MSS HTA to pol Mutations Associated with Resistance to RT Inhibitors. An MSS HTA can be developed rapidly for different target sequences. As an example, we generated an MSS
(A)
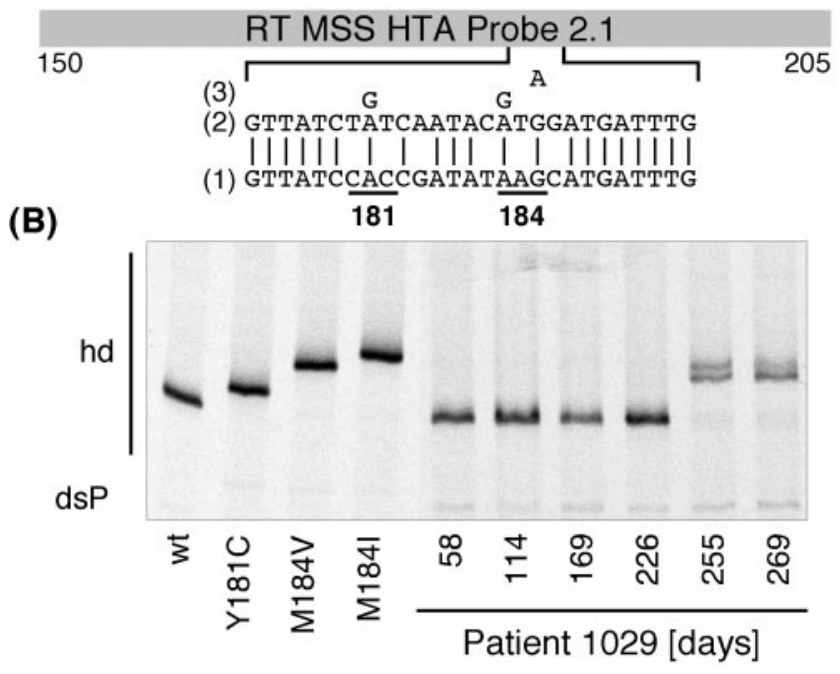

Fig. 6. Development of an RT MSS HTA. $(A)$ Sequence of the RT MSS HTA probe 2.1 (1) aligned to Hxb-2r wild-type sequence; (2) base changes involved in resistance (3) are shown above. $(B)$ Mobility of the radioactively labeled probe when annealed to three PCR products containing resistance mutations in comparison with the mobility of wild type (wt). In addition, plasma RNA of Patient 1029 who started 3TC therapy at day 215 was subjected to an MSS HTA analysis shown on the same gel. hd, heteroduplex; dsP, double-stranded probe.

HTA probe that targets nucleotide changes at positions 181 and 184 of the RT coding domain pol. Changes at these positions have been shown to confer resistance to nevirapine and $2^{\prime}, 3^{\prime}$ dideoxy-3'-thiacytidine (3TC), respectively $(24,25)$. The RT MSS probe 2.1 spans codons 150 through 205 of the RT coding domain and contains 6 nucleotide changes (Fig. $6 \mathrm{~A}$ ). It shows differential sensitivity to the three targeted mutations in the two positions (Fig. 6B). This probe was applied to investigate the evolution of resistance to $3 \mathrm{TC}$ in an individual. Rapid turnover of populations after the addition of 3TC to the existing drug regimen on day 215 is evident (Fig. $6 B$ ). Sequence analysis confirmed that the observed mobility shift correlated with the emergence of a base change at position 184.

\section{Discussion}

The protease and RT MSS HTAs introduced here represent an improvement over the different techniques used up to this point for the investigation into the population dynamics of viral genotypes. This assay is distinct from the regular HTA because of its sensitivity to single base changes and the partial selectivity for predefined mutations. Although this selectivity is desirable, it also limits the MSS HTA to systems with known mutations. In contrast to the universal heteroduplex generator probes described previously, which were designed to detect single point mutations, MSS probes can detect multiple mutations in a genome, and the presence of multiple linked mutations in a single genome typically reduces further the migration in the gel over single point mutations. This feature of HTA allows the resolution of populations that differ by the number of mutations. Moreover, compared with other assays used in population dynamics, such as single-strand conformational polymorphism and sequencing of clones, MSS HTA is simpler and does not require specialized equipment. The longer initial setup time of MSS HTA, compared with the sequencing of clones, is recovered by the rapid analysis of large numbers of samples. Even though the MSS HTA cannot completely obviate sequencing methods, it can serve to focus sequencing efforts to a subset of relevant 
samples while providing accurate information about the frequencies of different genotypic species within the viral population. The ability of MSS HTA to resolve discrete complex genotypic species distinguishes it from other simple assays, such as the line probe assay, PCR-based assays, or bulk sequencing.

The sensitivity of the pro probe is largely restricted to the targeted mutations. Mutations in neighboring positions, however, can cause fortuitous mobility shifts (Fig. 2). We do not expect this to be a major pitfall, because most of the adjacent positions in pro appear to be conserved in a set of unrelated pro sequences retrieved from a sequence database (22). The narrow sensitivity distribution allows the selective detection of resistance-relevant mutations without significant interference from background mutations.

The heteroduplex of the pro probe and the wild-type pro PCR product has a mobility of 0.90 because of the design of the MSS HTA probe. The lowest observed mobility for the pro HTA in this study was 0.73 . Because bands with mobilities differing by 0.01 are resolved, we theorize that up to 18 populations could be resolved in a single sample. As the number of possible combinations of the six target mutations exceeds the theoretical resolution, it is impossible to assign a single genotype to each band. For this and other reasons discussed earlier, the MSS HTA correlates with the phenotype of viral populations only in the sense that there is a correlation between a greater than 2.5 -fold reduction in sensitivity in the presence of at least one of the targeted mutations and a reduced mobility ratio (Fig. 4). The inability to assign a specific genotype to MSS HTA bands, and the imperfection of the correlation between HTA shift and phenotypic drug susceptibility, would seem to limit the usefulness of MSS HTA for cross-sectional analyses of pro evolution.

1. Stuyver, L., Wyseur, A., Rombout, A., Louwagie, J., Scarcez, T., Verhofstede, C., Rimland, D., Schinazi, R. F. \& Rossau, R. (1997) Antimicrob. Agents Chemother. 41, 284-291.

2. Anderson, B. D., Shirasaka, T., Kojima, E., Yarchoan, R. \& Mitsuya, H. (1994) Antiviral Res. 25, 245-258.

3. Piatek, A. S., Tyagi, S., Pol, A. C., Telenti, A., Miller, L. P., Kramer, F. R. \& Alland, D. (1998) Nat. Biotechnol. 16, 359-363.

4. Abravaya, K., Carrino, J. J., Muldoon, S. \& Lee, H. H. (1995) Nucleic Acids Res. 23, 675-682.

5. Frenkel, L. M., Wagner, L. E., II, Atwood, S. M., Cummins, T. J. \& Dewhurst, S. (1995) J. Clin. Microbiol. 33, 342-347.

6. Larder, B. A., Kohli, A., Kellam, P., Kemp, S. D., Kronick, M. \& Henfrey, R. D. (1993) Nature (London) 365, 671-673.

7. Bazar, L. S., Collier, G. B., Vanek, P. G., Siles, B. A., Kow, Y. W., Doetsch, P. W., Cunningham, R. P. \& Chirikjian, J. G. (1999) Electrophoresis 20, 1141-1148.

8. Eastman, P. S., Boyer, E., Mole, L., Kolberg, J., Urdea, M. \& Holodniy, M. (1995) J. Clin. Microbiol. 33, 2777-2780.

9. Delwart, E. L., Sheppard, H. W., Walker, B. D., Goudsmit, J. \& Mullins, J. I. (1994) J. Virol. 68, 6672-6683.

10. Nelson, J. A., Fiscus, S. A. \& Swanstrom, R. (1997) J. Virol. 71, 8750-8758.

11. Sullivan, D. G., Wilson, J. J., Carithers, R. L., Jr., Perkins, J. D. \& Gretch, D. R. (1998) J. Virol. 72, 10036-10043.

12. Ping, L. H., Nelson, J. A., Hoffman, I. F., Schock, J., Lamers, S. L., Goodman, M., Vernazza, P., Kazembe, P., Maida, M., Zimba, D., et al. (1999) J. Virol. 73, 6271-6281.

13. Wood, N., Tyfield, L. \& Bidwell, J. (1993) Hum. Mutat. 2, 131-137.
In longitudinal studies, the aforementioned shortcomings of the assay are less important because a change in the mobility of bands relative to the populations at baseline is measured over time rather than the absolute mobility of bands at a given time. We applied the pro and the RT MSS HTA to longitudinal samples to illustrate the behavior of the assays. In the case of pro, we showed the stepwise accumulation of resistance mutations in the viral populations correlating with successively slower-migrating HTA bands in vitro and in vivo. Moreover, it was observed that several populations frequently coexisted and that the turnover of viral populations was rapid. Similar results were obtained for the RT MSS HTA in vivo. Specifically, we saw the emergence of resistance to 3TC 40 days after therapy start.

The data presented here suggest that the MSS HTA will be a useful tool for the investigation of population dynamics. We can take advantage of this technology to investigate not only population dynamics in vivo, but also viral fitness in competition cultures in vitro. In addition, the capabilities of both the pro and RT probes can be expanded. The technology described here can be deployed likewise to investigate sequence evolution in other regions of HIV-1 and in other organisms. Possible examples include the evolution of the human ras gene in oncogenesis or bacterial genes involved in pathogenesis or resistance.

We thank Drs. J. Nelson and A. Kaplan for critical review of the manuscript. Plasma samples were graciously provided by Dr. D. Kempf at Abbott Laboratories. This work was supported by National Institutes of Health Grant RO1 AI25321 (to R.S.) and Pediatric AIDS Foundation fellowships (to W.R. and E.L.S).

14. Williams, D. L., Spring, L., Gillis, T. P., Salfinger, M. \& Persing, D. H. (1998) Clin. Infect. Dis. 26, 446-450.

15. Stoerker, J., Hurwitz, C., Rose, N. C., Silberstein, L. E. \& Highsmith, W. E. (1996) Clin. Chem. 42, 356-360.

16. Hirsch, M. S., Conway, B., D’Aquila, R. T., Johnson, V. A., Brun-Vezinet, F., Clotet, B., Demeter, L. M., Hammer, S. M., Jacobsen, D. M., Kuritzkes, D. R., et al. (1998) J. Am. Med. Assoc. 279, 1984-1991.

17. Ratner, L., Fisher, A., Jagodzinski, L. L., Mitsuya, H., Liou, R. S., Gallo, R. C. \& Wong-Staal, F. (1987) AIDS Res. Hum. Retroviruses 3, 57-69.

18. Kunkel, T. A., Bebenek, K. \& McClary, J. (1991) Methods Enzymol. 204, 125-139.

19. Vaillancourt, M., Irlbeck, D., Smith, T., Coombs, R. W. \& Swanstrom, R. (1999) AIDS Res. Hum. Retroviruses 15, 355-363.

20. Petropoulos, C. J., Parkin, N. T., Limoli, K. L., Lie, Y. S., Wrin, T., Huang, W., Tian, H., Smith, D., Winslow, G. A., Capon, D. J. \& Whitcomb, J. M. (2000) Antimicrob. Agents Chemother. 44, 920-928.

21. Loeb, D. D., Swanstrom, R., Everitt, L., Manchester, M., Stamper, S. E. \& Hutchison, C. A., III. (1989) Nature (London) 340, 397-400.

22. Shafer, R. W., Jung, D. R., Betts, B. J., Xi, Y. \& Gonzales, M. J. (2000) Nucleic Acids Res. 28, 346-348.

23. Cameron, D. W., Heath-Chiozzi, M., Danner, S., Cohen, C., Kravcik, S., Maurath, C., Sun, E., Henry, D., Rode, R., Potthoff, A. \& Leonard, J. (1998) Lancet 351, 543-549.

24. Gao, Q., Gu, Z., Parniak, M. A., Cameron, J., Cammack, N., Boucher, C. \& Wainberg, M. A. (1993) Antimicrob. Agents Chemother. 37, 1390-1392.

25. Richman, D. D., Havlir, D., Corbeil, J., Looney, D., Ignacio, C., Spector, S. A., Sullivan, J., Cheeseman, S., Barringer, K., Pauletti, D., et al. (1994) J. Virol. 68, 1660-1666. 